\title{
La elección de destino según las características de las comunidades autónomas de residencia
}

\author{
Tamara de la Mata \\ Investigadora asociada al Departamento de Análisis Económico y del Instituto L. R. Klein/CEPREDE. \\ Universidad Autónoma de Madrid. \\ 28049 Canto Blanco. Madrid. Tel. y fax: 91497867 \\ tamara.delamata@uam.es
}

\section{RESUMEN}

En este artículo se analizan mediante un logit multinomial los factores que afectan a la decisión de destino de los residentes en España según la Encuesta Movilia 2007. A partir de los resultados obtenidos para el total de la muestra y para los residentes de diferentes comunidades autónomas, se pretende comparar si la propensión a viajar fuera de las propias regiones de origen es mayor para algunas comunidades autónomas que para otras o en qué medida estas diferencias se deben a cuestiones geográficas, culturales o de otro tipo de factores. Se han obtenido resultados concluyentes a favor de la importancia de controlar cierta heterogeneidad según las comunidades autónomas de residencia de los individuos.

Palabras clave: elección de destino; turismo de residentes; modelo logit multinomial.

Clasificación JEL: R12 - Size and Spatial Distributions of Regional Economic Activity; Interregional Trade. L8 - Industry Studies: Services. L83 - Sports; Gambling; Recreation; Tourism. 


\section{INTRODUCCIÓN}

Con las reducciones de los costes de transporte, los patrones turísticos también cambian. Cada vez es más fácil y barato elegir como destino un lugar más exótico o lejano. Adicionalmente, la internacionalización de las relaciones económicas, favorece el aumento de los viajes al extranjero por motivos profesionales. Sin embargo, el turismo y los movimientos de las personas siguen teniendo un fuerte carácter regional, y a nivel nacional es importante destacar que los viajes cortos, de fin de semana o incluso las excursiones siguen teniendo un peso relativo muy importante.

Según la Cuenta Satélite del Turismo en el año 2006 del total de consumo turístico interior $^{1}$ en España, el $44 \%$ es consumo turístico de los hogares, y el $43 \%$ el receptor. Teniendo en cuenta la importancia de España como destino turístico a nivel mundial, podemos hacernos una idea de la relevancia que tienen los viajes de los nacionales para el total de la economía.

A pesar de la importancia de los movimientos interiores, la literatura se ha centrado más en los análisis de la demanda turística a partir de datos agregados como un fenómeno de movilidad internacional. Para una revisión de la literatura ver Witt y Witt (1995), Lim (1999), Song y Li (2008).

En el caso de España, algunos autores han estimado y analizado el turismo nacional de manera agregada desde diferentes enfoques (Largo Jiménez, 1976; Usach Domingo, 1998; Cañada, 2002; Millán Escriche, 2004; Aznar y Nicolini, 2007; Llano y de la Mata, 2009; entre otros).

Sin embargo, no son tan frecuentes los artículos en los que se han utilizado micro datos. En primer lugar, Alegre y Pou (2004) mediante un modelo logit aplicado a los micro datos de la Encuesta de Presupuestos Familiares para el período 1985-1996 analizan los factores que afectan a la decisión de viajar o no por motivos de ocio. En segundo lugar, un amplio grupo de artículos se centran en los determinantes de la duración de la estancia, como los de Alegre y Pou (2006); Martínez-García y Raya (2008); Artal et al (2010).

1 Según la metodología de la Cuenta Satélite del Turismo (INE, 2004), se considera como turismo interior las actividades turísticas efectuadas tanto por residentes (turismo interno) como por no residentes (turismo receptor) dentro del territorio económico nacional, definiendo como Turismo las actividades que realizan las personas durante sus viajes y estancias en lugares distintos al de su entorno habitual, por un período de tiempo consecutivo inferior a un año, con fines de ocio, por negocios u otros motivos. 
En relación a la elección de un destino turístico, Riera (2000) aplica un modelo de elección discreta basado en las funciones de utilidad, aplicándolo a los turistas en los espacios naturales protegidos de la isla de Mallorca. Sin embargo, por primera vez en Nicolau y Más (2005) se estima un modelo de elección discreta sobre las elecciones del destino turístico de los españoles. Analizan un proceso de elección de destino que incluye tres decisiones básicas: realizar o no un viaje, ir al extranjero o elegir un destino nacional y por último realizar un viaje a un único destino o elegir un destino múltiple. En Nicolau y Más (2006) se propone que el efecto de la distancia y los precios como factores determinantes de la elección de un destino determinado, queda moderado por los motivos que los individuos tengan para viajar. Finalmente, en Nicolau y Más (2008) se propone un proceso de toma de decisión del tipo de destino multietápico: realizar o no un viaje, elegir un destino costero o de interior, y finalmente, elegir un destino urbano o no. En los tres casos utilizan datos de la Encuesta de «Comportamiento de los españoles ante las vacaciones» del Centro de Investigaciones Sociológicas. Son datos de 1995 y cuentan con una muestra de 3.781 individuos.

Ninguno de estos artículos se ha centrado en analizar cómo las características geográficas o culturales de las provincias o comunidades autónomas (CCAA) de residencia de los individuos, afectan a la elección de un tipo de destino determinado. El objetivo de este trabajo es analizar en qué medida las características de los individuos afectan al tipo de destino que elijan, pero principalmente, en qué medida las provincias y CCAA en las que residen, su situación geográfica y peculiaridades culturales afectan a la elección del tipo de destino. Para ello utilizaremos los datos de la Encuesta de Movilia 2007 recogidos a nivel provincial, elaborada por el Ministerio de Fomento. A través de la aplicación de un logit multinomial, confirmamos algunos resultados sobre cómo algunas características como el tamaño del municipio de residencia puede afectar a la elección de un determinado destino turístico. En segundo lugar, obtendremos importantes resultados sobre cómo las peculiaridades geográficas o culturales de las CCAA de residencia puede afectar a las preferencias por uno u otro tipo de destino. En este sentido, encontramos por ejemplo, que el hecho de residir en una comunidad o provincia fronteriza con otro país favorezca la elección del extranjero como destino o que el tamaño de las comunidades afecte a la elección del destino. Por otro lado, encontramos que en determinadas ocasiones una misma característica geográfica o cultural produce efectos diferentes. Este sería el caso de la probabilidad de viajar al extranjero para los residentes en Canarias y Baleares o la probabilidad de viajar a otras comunidades autónomas en Cataluña, Galicia o el País Vasco.

La estructura del trabajo es la siguiente: en el siguiente apartado introduciremos las teorías de la utilidad aleatoria y el modelo logit multinomial que vamos a utilizar. En 
el tercer apartado haremos un análisis descriptivo de los tipos de destinos elegidos por los residentes en España según la Encuesta Movilia 2007. En el cuarto apartado, explicaremos la metodología seguida y los resultados obtenidos con el modelo de elección discreta. Finalmente, se incluyen unas breves conclusiones y extensiones del artículo para futuras investigaciones.

\section{LA TEORÍA DE LA UTILIDAD ALEATORIA Y EL MODELO DE ELECCIÓN DISCRETA}

Los modelos de elección discreta tratan de estimar la probabilidad de elegir una alternativa frente a otras en función de las características tanto de las alternativas como de los individuos. Si bien tienen como punto de partida la teoría neoclásica de las características de Lancaster (1966), en la que los bienes proporcionan utilidad a los individuos a través de sus características, se diferencian de ésta en que asumen «racionalidad limitada» (Simon, 1955). Por el contrario, según el enfoque basado en las teorías de producción doméstica (Becker, 1965) serían las restricciones en tiempo y dinero del individuo las que determinarían un reparto de los recursos (tiempo y dinero) del individuo hacia bienes que les proporcionen utilidad. Estas teorías se han aplicado al caso del turismo en los trabajos mencionados anteriormente mediante diferentes tipos de modelos econométricos de elección discreta (Baltas, 2006).

Dentro de las teorías de elección discreta se suele distinguir entre las teorías de utilidad constante -en las que la utilidad es constante y las reglas de decisión son estocásticas-, y las teorías de utilidad aleatoria con reglas deterministas. Dentro de este segundo grupo, el hecho de que la utilidad sea aleatoria implica que ésta tenga cierta variabilidad debido a la falta de información del individuo que decide o del investigador que analiza la información. Este enfoque ha sido desarrollado por Thursone (1927, 1945), Mc Fadden (1974) y Mansky (1977).

La teoría de Mc Fadden (1974) y Mansky (1977) incluye un componente aleatorio en la función de utilidad del individuo debido a la imposibilidad del analista por obtener toda la información relativa al proceso de elección al que se enfrenta el individuo. Así la función de utilidad del individuo i está formada por un componente determinista conocido y un componente aleatorio (no observado). De esta forma la probabilidad de que el individuo i elija la alternativa j dependerá de la utilidad que ésta le reporte (eq.

(1)) que estará compuesta en su parte determinista tanto por atributos de las alternativas $\left(\mathrm{x}_{\mathrm{j}}\right)$ como por características de los individuos $\left(\mathrm{z}_{\mathrm{i}}\right)$. 


$$
U_{i j}=V_{i j}+\varepsilon_{i j}=V\left(x_{j}, z_{i}\right)+\varepsilon_{i j}
$$

En el caso de que la decisión sea por ejemplo, realizar o no un viaje, el individuo lo realizará si la utilidad neta es positiva. Si la elección es entre un conjunto J de alternativas, por ejemplo destinos o modos de viaje, la probabilidad de que el individuo i elija la alternativa $\mathrm{j}$ será mayor cuanto mayor sea la probabilidad de que la utilidad que le reporte la alternativa $\mathrm{j}$ sea mayor que la utilidad que le reporte el resto de alternativas. De tal forma que la probabilidad de que el individuo i escoja la alternativa j será:

$$
P_{j}(i)=P\left(V_{i j}+\varepsilon_{i j}>V_{i m} \varepsilon_{i m}\right) \quad \text { para todo } \mathrm{m} \in \mathrm{J}, \mathrm{m} \neq \mathrm{j}
$$

Si la perturbación aleatoria sigue una distribución normal, estaríamos hablando de un modelo probit multinomial. Suponiendo que la perturbación aleatoria se distribuye idéntica e independiente siguiendo una distribución logística (Gumbel I) y que la parte determinista de la utilidad $V_{i j}$ se relaciona de forma lineal con las variables relativas al individuo $z_{i}$, estaremos hablando de un logit multinomial ${ }^{2}$. En un logit multinomial, se modelizan tantas ecuaciones como alternativas tiene el conjunto J. Sin embargo, es necesario identificar una categoría de referencia para que el modelo esté identificado, por lo que para cada variable obtendremos J-1 estimaciones de los parámetros (uno para cada alternativa excepto para la categoría de referencia). De tal forma que manteniéndose todo lo demás constante, un incremento unitario en una variable determinada aumenta (si el signo del coeficiente es positivo) o disminuye (si el signo del coeficiente es negativo), el logaritmo del ratio de probabilidades de cada una de las alternativas frente a la alternativa de referencia. Si la alternativa de referencia es la alternativa $m$, el modelo vendrá dado por:

$$
\begin{array}{ll}
P_{m}(i)=\frac{1}{1+\sum_{j=1}^{J} \exp \left(\beta_{j}{ }^{\prime} z_{j}\right)} & \text { para todo } \mathrm{j} \in \mathrm{J}, \mathrm{j} \neq \mathrm{m} \\
P_{j}(i)=\frac{\exp \left(\beta_{j}{ }^{\prime} z_{j}\right)}{1+\sum_{j=1}^{J} \exp \left(\beta_{j}{ }^{\prime} z_{j}\right)} & \text { para todo } \mathrm{j} \in \mathrm{J}, \mathrm{j} \neq \mathrm{m}
\end{array}
$$

${ }^{2}$ Si se incluyeran también variables relativas a las alternativas nos encontraríamos ante un logit condicional. Ante la falta de datos para las alternativas no elegidas se ha preferido optar por un logit multinomial y aproximar las restricciones temporales y económicas a partir de variables referentes a los individuos. 
Para estimar los coeficientes de este modelo se utiliza el método de máxima verosimilitud, tal que la función de máxima verosimilitud es:

$$
\text { h } L=\sum_{i=1}^{n} \sum_{j=1}^{J} d_{i j} \text { h } P_{j}(i)
$$

Donde $d_{i j}=1$ si el individuo i elige la alternativa j y 0 en otro caso.

\section{ANÁLISIS DESCRIPTIVO}

Existen diversas fuentes estadísticas dedicadas al estudio de los movimientos de los españoles. En nuestro caso hemos decidido utilizar la encuesta Movilia 2007 (Ministerio de Fomento, 2007) en la que se recoge información de carácter socio-económico de los residentes en las viviendas familiares en España, sus viajes y desplazamientos. En este momento, se encuentran disponibles Movilia 2000 y 2006 sobre movilidad cotidiana y Movilia 2001 y 2007 (Ministerio de Fomento, 2007) sobre viajes de mayor distancia.

En Movilia 2007 se recoge información de unos 35.000 viajes, pudiendo disponer de los microdatos sobre cada viaje, con información sobre la provincia de residencia del individuo y de origen viaje, el tamaño del municipio de residencia, el sexo de la persona entrevistada, el grupo de edad, actividad y estudios, el motivo del viaje, los modos de transporte utilizados, la distancia y duración del viaje y el tipo de provincia y comunidad autónoma de destino. La encuesta recoge diferentes tipos de viajes:

- Viajes a más de $50 \mathrm{~km}$ de distancia del lugar de origen (excluyendo viajes a centros de estudio o trabajo).

- Viajes a más de $50 \mathrm{~km}$ realizados a centros de estudio o trabajo.

- Viajes a menos de $50 \mathrm{~km}$ en los que se realiza al menos una pernoctación fuera del lugar de residencia.

Hemos eliminado de nuestra muestra los viajes de más de $50 \mathrm{~km}$ a centros de trabajo y estudio, ya que siguiendo la metodología de la Cuenta Satélite del Turismo (INE, 2004), los desplazamientos a los lugares de trabajo se consideran entorno habitual, aunque se encuentren alejados del domicilio. Este tipo de viajes únicamente representaba el $3 \%$ del total de la muestra.

La encuesta de Movilia 2007 presenta algunas limitaciones como que es una encuesta dirigida a los individuos, por lo que no recoge información sobre los hogares: número de personas, renta total disponible del hogar, número de hijos, etc. Por lo tanto, consideraremos a cada persona de forma individual. Adicionalmente, si bien es cierto 
que recoge el lugar de residencia a nivel provincial, no está disponible la provincia de destino del viaje. Sin embargo, sí se recoge si el destino elegido es la provincia de residencia, una limítrofe, si pertenece a la CCAA de residencia o no, o si el destino elegido es el extranjero.

A continuación vamos a realizar un análisis descriptivo de algunas de las variables recogidas en la encuesta de Movilia 2007 según el tipo de destino elegido.

\subsection{Tamaño del municipio}

Se ha comprobado el efecto positivo del mayor tamaño del municipio de residencia en la probabilidad de realizar un viaje (Dardis et al, 1981; Dardis et al, 1994; Cai, 1998, Alegre y Pou, 2004). Este efecto es debido a dos factores: la necesidad de «escapar» de las grandes urbes y la mayor disponibilidad de oportunidades. De forma similar, el mayor tamaño de los municipios, puede favorecer el hecho de elegir un destino más alejado de la provincia de residencia.

En nuestro caso, los residentes en municipios de mayor tamaño (6\% del total) eligen en menor proporción viajar a su provincia de residencia, mientras que prefieren otras provincias de su comunidad autónoma. Mientras tanto, los residentes en municipios más pequeños realizan más viajes dentro de su propia provincia $(35 \%$ frente al $19 \%$ de los municipios de mayor tamaño), como vemos en el Gráfico 1. Los residentes en municipios de mayor tamaño (> 500.000 habitantes) realizan incluso más viajes a provincias limítrofes de la misma comunidad o a otras provincias de otras regiones que a su propia provincia. Por lo tanto, esperamos una relación positiva entre el tamaño del municipio de residencia y la probabilidad de que se elija como destino una provincia o incluso una comunidad diferente a la de residencia. 
GRÁFICO 1.-Distribución de los viajes según el tamaño del municipio de residencia

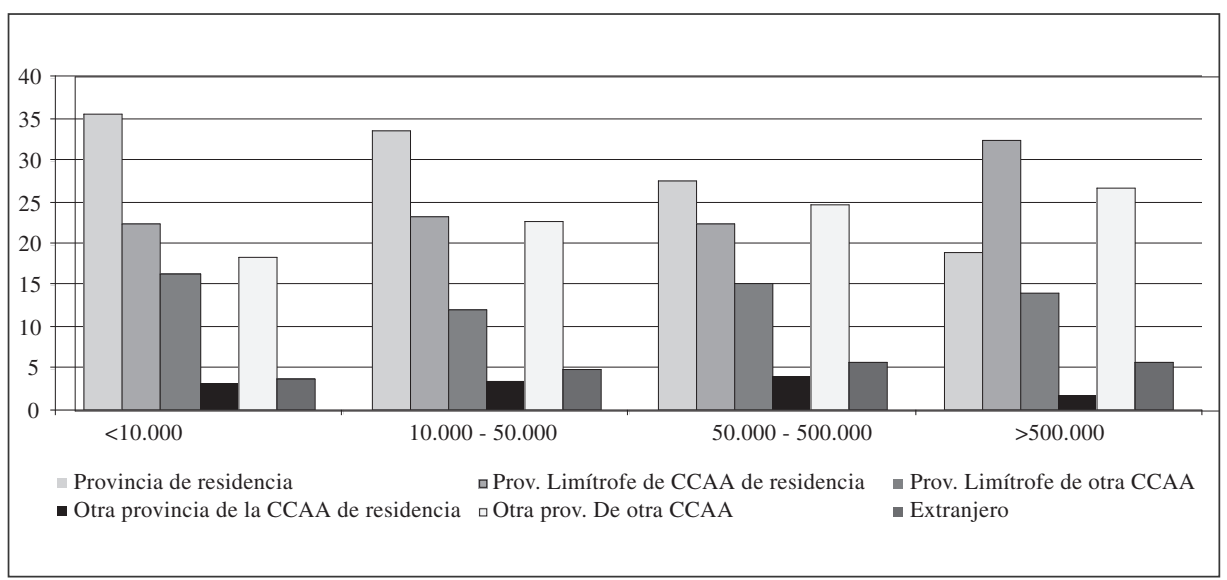

Fuente: Movilia 2007 (Ministerio de Fomento)

\subsection{Lugar de residencia}

El lugar de residencia puede afectar de tal forma que exista cierta heterogeneidad a la hora de elegir un destino por un motivo geográfico, cultural, etc.

Respecto a este punto podemos pensar por ejemplo, que el hecho de residir en las islas hace que se realicen un menor número de viajes, pero que una vez que un individuo se ha decidido a realizar un viaje, elija lugares más lejanos. Mientras que para los residentes en Ceuta y Melilla puede resultar más costoso realizar un viaje a la península que al continente africano dada su situación geográfica. Es posible que los residentes en algunas comunidades con particularidades culturales como una lengua cooficial, tenga preferencia por su propia cultura. Imm Ng et al (2007) apuntan la importancia de la inclusión de variables de carácter social y cultural en los análisis. Según señalan el hecho de que exista una mayor similitud cultural entre el lugar de residencia de un individuo y un posible destino turístico, aumenta la probabilidad de que el individuo elija ese destino (Basala y Klenosky, 2001; Henderson, 2003). Por lo tanto, es posible que el hecho de tener ciertas peculiaridades culturales aumente la preferencia por la CCAA de residencia y que, por consiguiente, se realicen proporcionalmente más viajes dentro de la propia región que al resto de España.

Es necesario señalar el efecto que el diferente tamaño de las diferentes comunidades tiene sobre la probabilidad de elegir como destino la CCAA de residencia o no. Es decir, para 
los residentes en comunidades de gran extensión como Andalucía o Castilla y León será más fácil desplazarse dentro de su comunidad, mientras que para los residentes en comunidades uniprovinciales será más probable que elijan un destino fuera de su comunidad.

GRÁFICo 2.-Viajes a la CCAA de residencia y a otra sobre el total por CCAAs

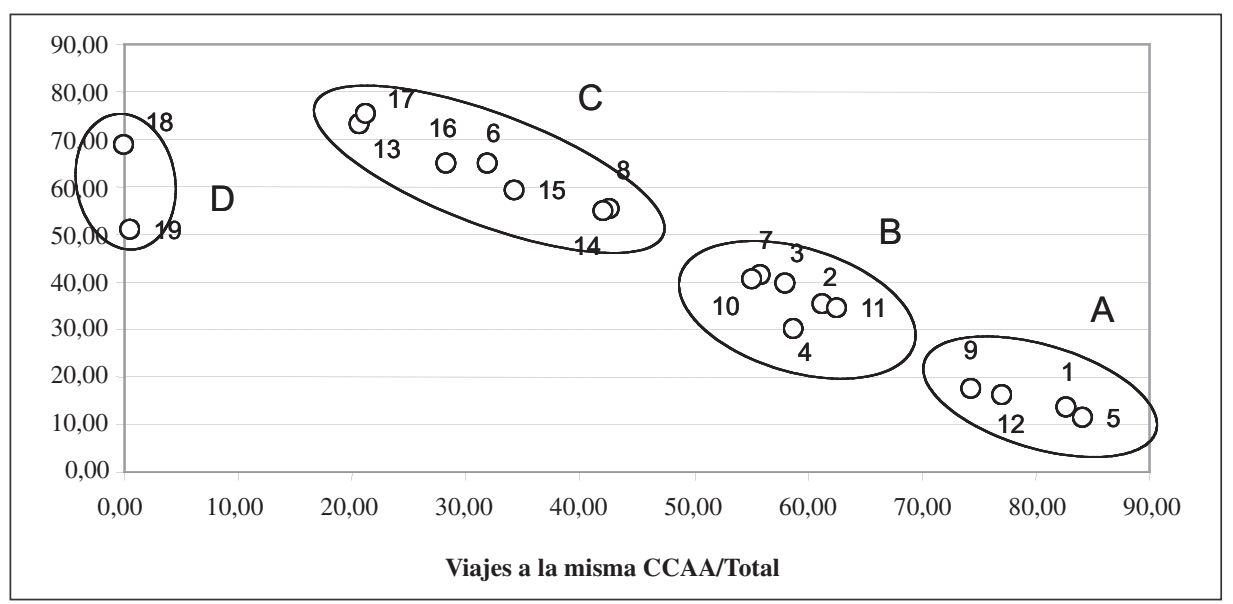

Fuente: Movilia 2007 (Ministerio de Fomento).

\begin{tabular}{|lll|lll|rll|lll|}
\hline 1 & A & Andalucía & 6 & C & Cantabria & 11 & B & Extremadura & 16 & C & País Vasco \\
2 & B & Aragón & 7 & B & Castilla y León & 12 & A & Galicia & 17 & C & La Rioja \\
3 & B & Asturias & 8 & C & $\begin{array}{l}\text { Castilla-La } \\
\text { Mancha }\end{array}$ & 13 & C & $\begin{array}{l}\text { Com. de } \\
\text { Madrid }\end{array}$ & 18 & D & Ceuta \\
4 & B & Baleares & 9 & A & $\begin{array}{l}\text { Cataluña } \\
\text { Com. }\end{array}$ & 14 & C & R.de Murcia & 19 & D & Melilla \\
5 & A & Canarias & 10 & B & $\begin{array}{l}\text { Com. } \\
\text { Valenciana }\end{array}$ & 15 & C & Navarra & & & \\
\hline
\end{tabular}

Con el objetivo de realizar un primer análisis exploratorio, en el Gráfico 2 se ha representado un gráfico de dispersión que nos muestra para cada CCAA el porcentaje de viajes realizados dentro de su misma comunidad respecto del total con el porcentaje de viajes realizados a otra $\mathrm{CCAA}^{3}$. En el Gráfico 2 se distinguen 4 grupos diferenciados.

${ }^{3}$ El porcentaje de viajes a la comunidad de residencia más el de viajes a otras comunidades es inferior al $100 \%$ puesto que son porcentajes sobre el total de viajes. El resto son viajes al extranjero. 
En el Grupo A, encontramos las comunidades de Andalucía, Canarias, Galicia y Cataluña. Los residentes en estas comunidades autónomas realizan alrededor del $80 \%$ de sus viajes a su comunidad de residencia mientras que únicamente realizan entre el 10 y el $20 \%$ de sus viajes a otras comunidades. Este comportamiento podría venir explicado por el gran tamaño y oferta turística local, el hecho de que son regiones periféricas o sus especificidades lingüísticas y culturales. Sin embargo, existen otras comunidades de gran tamaño como Castilla y León o que son islas como Baleares o con peculiaridades culturales como el País Vasco, que no están en este mismo grupo.

En segundo lugar, el Grupo B está compuesto por Aragón, Asturias, Baleares, Castilla y León, Comunidad Valenciana y Extremadura que realizan entre el 55 y el $65 \%$ de los viajes a la comunidad en la que residen y entre el 30 y el $40 \%$ a otras comunidades. Estas comunidades tienen un comportamiento similar al comportamiento del total nacional (58\% de los viajes a la comunidad y $37 \%$ a otras comunidades).

Adicionalmente, encontramos un amplio grupo de comunidades (Grupo C) que realizan proporcionalmente menos viajes a su propia comunidad autónoma y más al resto. Este grupo está compuesto por Cantabria, Castilla La Mancha, Murcia, Navarra, Madrid, País Vasco y La Rioja. Es un grupo bastante heterogéneo, donde destaca la gran cantidad de comunidades uniprovinciales, puesto que en estos casos el proceso de decisión se limita a viajar a la CCAA de residencia o a otra.

Por último, encontramos a Ceuta y Melilla (Grupo D). Su reducido tamaño provoca que los residentes en las ciudades autónomas apenas realicen viajes dentro de su comunidad autónoma (0-1\%), sin embargo realizan un gran porcentaje de sus viajes al extranjero.

Para poder realizar un análisis origen-destino, en la Encuesta Movilia 2007 se ofrecen los datos del número total de viajes según la CCAA de residencia y de destino del viaje. En el Gráfico 3, las flechas señalan los destinos que representan más del $10 \%$ del total de viajes realizados desde cada CCAA. Es decir, la existencia de una flecha no implica que sean un flujo intenso a nivel nacional, sino que son destinos importantes para cada CCAA, teniendo en cuenta el número total de viajes que se producen desde ese origen. De esta forma, vemos la importancia de los viajes a las regiones vecinas para algunas CCAA como Madrid, País Vasco, Castilla León o Castilla La Mancha. 
GRÁFICo 3.-Principales destinos para cada CCAA

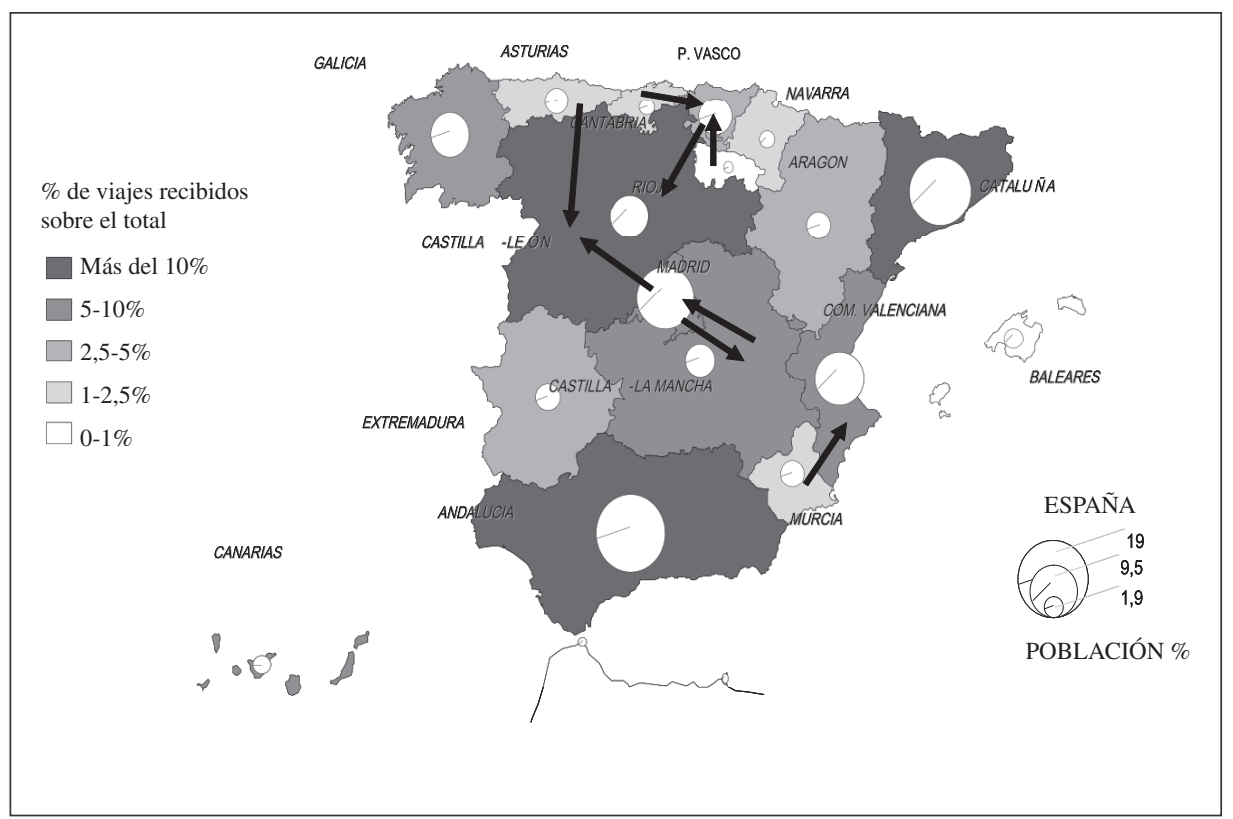

\subsection{Grupo de actividad}

A pesar de no disponer de variables de renta, podemos utilizar como proxy el grupo de actividad (Nicolau y Más, 2005; Walsh et al, 1992; Riera, 2000). En primer lugar, suponemos que los ocupados tienen una renta mayor, por lo que tendrán una menor restricción en la renta para viajar más lejos. Sin embargo, los viajes de los ocupados implican un mayor coste de oportunidad y una mayor restricción de tiempo para viajar (Ghez y Becker, 1975). En segundo lugar, el nivel de renta de los parados es bajo y además tenderán a ser cautelosos con sus gastos, por lo que su propensión a viajar será negativa (Deaton, 1997). Por la misma razón se supone que tenderán a elegir un destino más cercano que implique un menor coste. En tercer lugar, en el caso de los jubilados y de los estudiantes es necesario destacar que el hecho de disfrutar de ciertas subvenciones o descuentos en el precio al que deben hacer frente el resto de colectivos, reduce sus restricciones en la renta. 
GRÁFICO 4.-Distribución de los viajes según grupo de actividad.

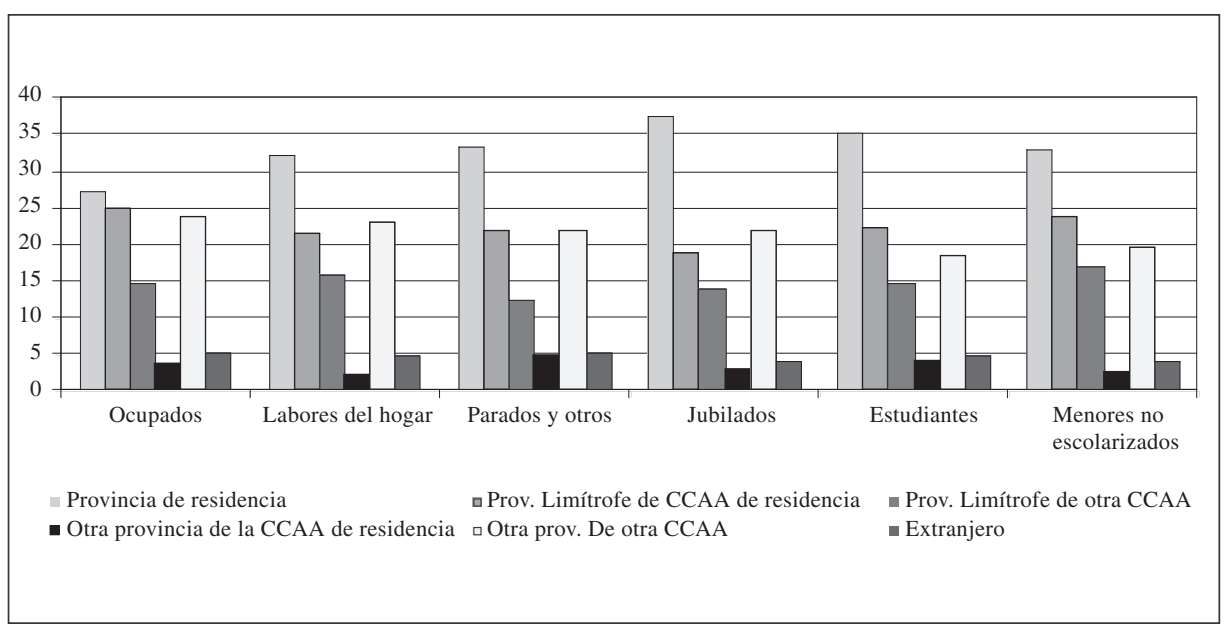

Fuente: Movilia 2007 (Ministerio de Fomento)

Tal y como vemos en el Gráfico 4, los ocupados tienden a viajar en menor proporción a su provincia de residencia, mientras que viajan más a provincias limítrofes dentro de su CCAA de residencia. Por el contrario, los jubilados realizan una mayor proporción de viajes dentro de su provincia de residencia.

\subsection{Nivel de estudio}

El hecho de ser universitario, puede estar relacionado con un mayor nivel de renta (Riera, 2000). Además, es posible que aumente la probabilidad de viajar más lejos porque los individuos con cierto nivel de estudios, tengan ciertos intereses culturales, o una menor barrera cultural a la hora de elegir destino.

Como vemos en el Gráfico 5, los universitarios tienden a viajar en una menor proporción dentro de la misma provincia en la que residen, mientras que tienen una mayor preferencia por el extranjero o provincias que no pertenezcan a la comunidad donde residen que los individuos con niveles de educación inferiores. En el caso de los universitarios, los viajes a provincias limítrofes superan a los que se realizan dentro de la misma provincia, mientras que en el caso de los individuos sin estudios los viajes a la misma provincia casi doblan a los de provincias limítrofes, dentro de la comunidad. 
GRÁFICo 5.-Distribución de los viajes según nivel de estudios

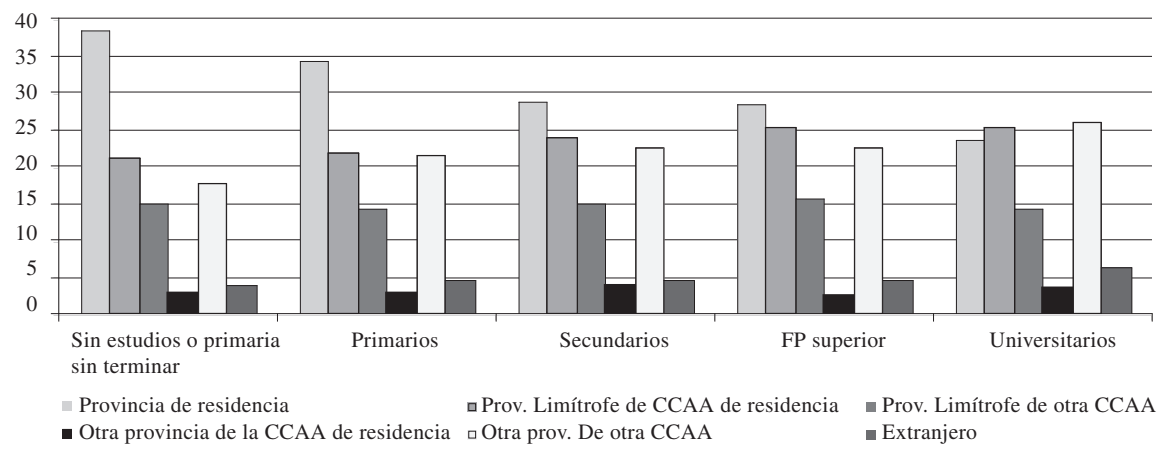

Fuente: Movilia 2007 (Ministerio de Fomento)

\subsection{Edad}

Tal y como se ha señalado en varios trabajos, el efecto de la edad en la probabilidad de viajar no es lineal (Walsh et al, 1992). El efecto de la edad puede variar en función de otras variables como la renta (Eymann y Ronning, 1997) o el ciclo vital (Lawson, 1991; Opperman, 1995; Collins y Tisdell, 2002) que hace que exista una relación en forma de $U$ invertida entre la probabilidad de viajar y la edad. Es posible pensar, que las mismas variaciones en las restricciones temporales y de renta o en las preferencias según la edad de los individuos que afectan a la probabilidad de viajar o no, se puedan encontrar en la probabilidad de elegir un destino determinado.

En el Gráfico 6, se puede ver cómo en nuestro caso encontramos una forma de U en la proporción sobre el total de los viajes a la provincia de residencia según la edad de los individuos, de tal forma que las personas de menor y mayor edad son las que, en una mayor proporción, eligen su provincia de residencia como destino de sus viajes. Esto se traduce en una ligera U invertida en la proporción de viajes a una provincia limítrofe perteneciente a la CCAA de residencia. Por el contrario, las personas de edades medias escogen el extranjero y otras provincias fuera de su comunidad como destino. 
Gráfico 6.-Distribución de los viajes según grupo de edad

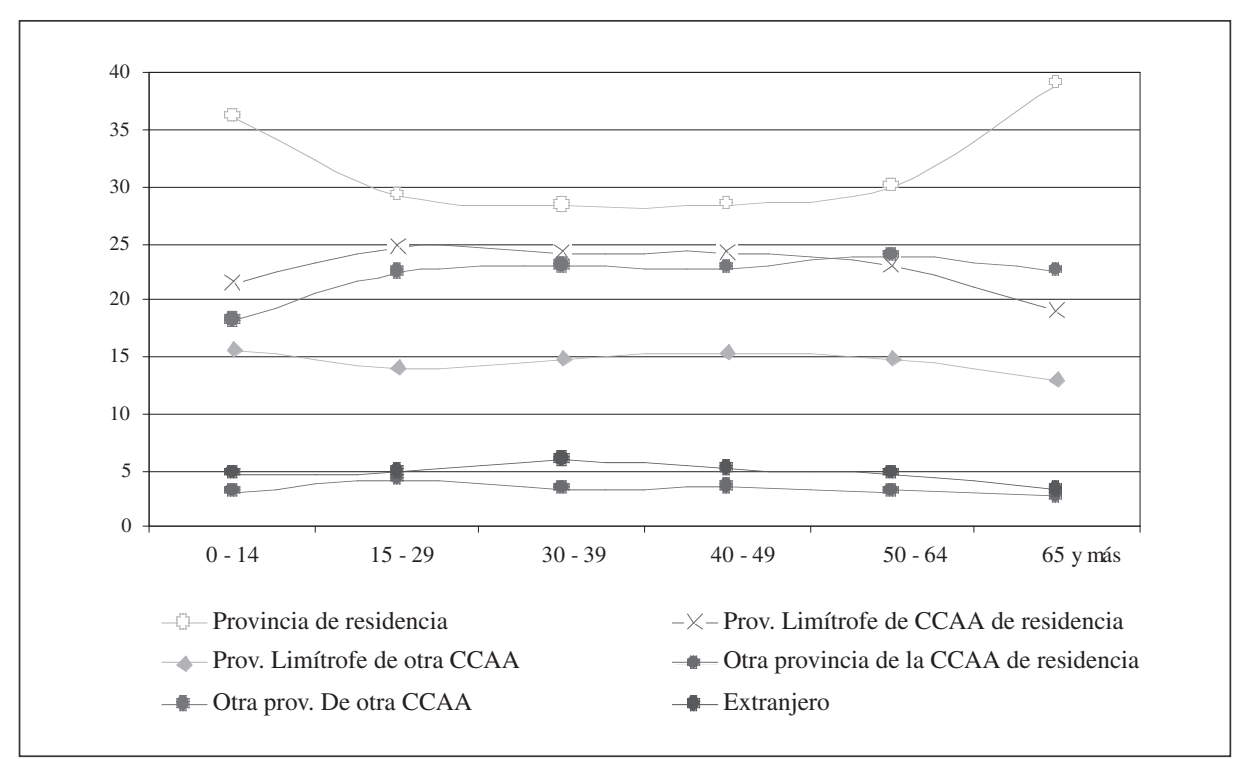

Fuente: Movilia 2007 (Ministerio de Fomento)

\subsection{Motivo del viaje}

Tal y como demuestran Nicolau y Más (2006) los propósitos con los que se realiza un viaje pueden moderar el efecto del precio y de la distancia como factores determinantes en la elección de un lugar determinado como destino turístico.

Según se ve en el Gráfico 7, los viajes cuyo propósito es el ocio tienen en mayor proporción un destino en el extranjero o a una provincia no limítrofe de otra comunidad que el resto de los viajes. En la Encuesta de Movilia 2007, los viajes por motivo de «Ocio» o de «Vacaciones» son aquellos realizados por diversión o descanso. La diferencia entre ambos está en que los viajes por «Ocio» tienen una duración inferior a 4 pernoctaciones, mientras que los viajes por motivo de «Vacaciones» tienen una duración superior a 4 pernoctaciones. Posiblemente el gran porcentaje de viajes al extranjero por motivos de ocio se ha visto favorecido por el desarrollo de las líneas aéreas de bajo coste. Por el contrario, los viajes a segunda residencia y a viviendas de familiares y amigos mayoritariamente se realizan a un destino nacional (los viajes a segunda residencia un $49 \%$ de las veces a la misma provincia). 


\section{GRÁFICo 7.-Distribución de los viajes según el motivo}

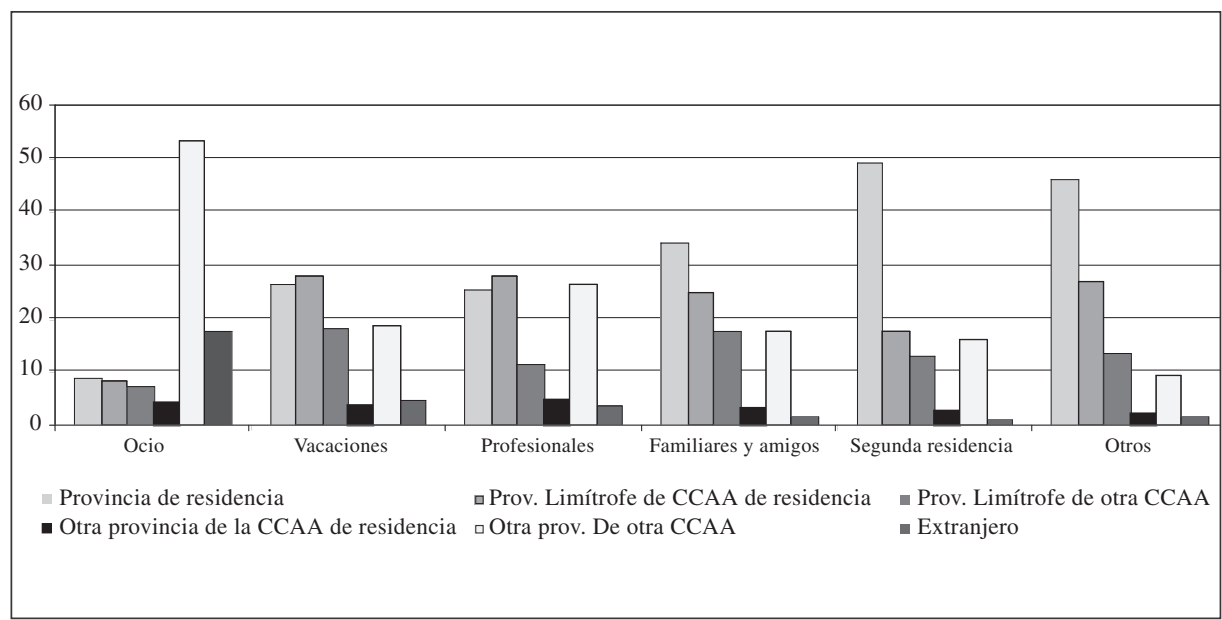

Fuente: Movilia 2007 (Ministerio de Fomento)

\section{ANÁLISIS MICROECONOMÉTRICO}

\subsection{Metodología}

Utilizando un modelo logit multinomial se han estimado dos especificaciones diferentes con el objetivo de comprobar cuáles son los factores que afectan a la elección de un determinado destino y en qué medida las características de unas y otras comunidades autónomas afectan a estas decisiones.

En la encuesta de Movilia 2007 no está disponible la provincia de destino del viaje. Sin embargo, tenemos el tipo de destino elegido. De esta forma, nuestra variable categórica que incluye las alternativas entre las que los individuos eligen son: viajar a la provincia de residencia (MP MC), a una provincia limítrofe de la CCAA de residencia (PL MC), a una provincia limítrofe de otra CCAA (PL OC), a una provincia no limítrofe de la comunidad de residencia (OP MC), a una provincia no limítrofe de otra comunidad (OP OC) o al extranjero (EX). Esta forma de definir el tipo de destino impide que para los residentes en las comunidades autónomas uniprovinciales podamos tener viajes a otra provincia de la misma comunidad. Del mismo modo, los residentes en las islas o en las ciudades autónomas no pueden elegir viajes a una provincia limítrofe sea de la misma o de otra CCAA. 
Incluimos como variables explicativas, algunos factores que pueden influir en la elección de un destino y que por tanto es necesario controlar. En primer lugar, como variable proxy de la renta, incluimos una interacción entre la dummy de ocupados y el nivel de estudio. De esta manera incluimos la relación entre educación y renta de tal forma que para el grupo de ocupados sin estudios le corresponderá el nivel de renta más bajo, mientras que los ocupados con estudios universitarios se corresponderá con el grupo de mayor nivel de renta. Incluimos las dummies que hacen referencia a la edad ${ }^{4}$, dejando a los mayores de 65 años como grupo de referencia y el tamaño del municipio de residencia, donde nos interesa especialmente el comportamiento de los municipios de mayor tamaño en relación a los más pequeños, por lo que el grupo de los municipios de menos de 10.000 habitantes serán nuestro grupo de control. Asimismo incluimos las variables ficticias relacionadas con el motivo por el que se realiza el viaje, incluyendo los viajes por ocio, vacaciones, motivos profesionales y visitar a familiares y amigos. Adicionalmente, incluimos como variable de control la variable duración que contiene el número de noches.

En primer lugar, estimamos el modelo incluyendo una dummy para las regiones uniprovinciales (cuniprov), otra para las ciudades autónomas (ceu_meli) y otra para los archipiélagos (islas) para controlar cómo pueden estar afectando sus características geográficas a sus decisiones sobre destinos. Hemos incluido también dos dummies ( $\boldsymbol{l i m \_ F r}$ y $\boldsymbol{l i m} \_$Por) que recogen a las provincias que son limítrofes tanto con Francia con Portugal, con el objetivo de controlar el posible efecto positivo sobre la probabilidad de viajar al extranjero siendo un residente en las provincias que comparten frontera con ambos países.

En una segunda especificación, incluimos una dummy para cada CCAA de residencia, excepto para las ciudades autónomas que debido a la falta de datos para estimar coeficientes diferentes para cada una de ellas para algunos tipos de viajes y su comportamiento similar, están recogidas en la variable ceu_meli. En este caso, dejamos la comunidad de Castilla y León como grupo de referencia por ser una comunidad de un gran tamaño con un comportamiento en la media (Tabla 2).

4 Tratando de buscar el comportamiento no lineal de la edad, se han hecho estimaciones que incluyeran una interacción de la edad y los niveles educativos o del grupo de actividad (como proxies del nivel de renta). Sin embargo, los resultados no han sido satisfactorios, por lo que hemos preferidos finalmente no incluirlas en la especificación presentada. 
En la siguiente tabla (Tabla 1) se muestra de forma resumida las variables utilizadas, así como la forma en que han sido codificadas y la categoría de referencia de cada una de ellas.

TABLA 1.- Variables utilizadas y categoría de referencia

\begin{tabular}{|c|c|c|}
\hline VARIABLES & CODIFICACIÓN & $\begin{array}{l}\text { CATEGORÍA } \\
\text { DE } \\
\text { REFERENCIA }\end{array}$ \\
\hline $\begin{array}{l}\text { Tipo de } \\
\text { destino. } \\
\text { Variable } \\
\text { categórica. }\end{array}$ & $\begin{array}{l}\text { MP MC: provincia de residencia. } \\
\text { PL MC: provincia limítrofe de la misma CCAA. } \\
\text { PL OC: provincia limítrofe de otra CCAA. } \\
\text { OP MC: provincia no limítrofe de la CCAA de } \\
\text { residencia. } \\
\text { OP OC: provincia no limítrofe de una CCAA diferente a } \\
\text { la de residencia. } \\
\text { EX: extranjero. }\end{array}$ & MP MC \\
\hline $\begin{array}{l}\text { Tamaño del } \\
\text { municipio de } \\
\text { residencia }\end{array}$ & $\begin{array}{l}\text { tama1: }<10.000 \text { habitantes. } \\
\text { tama2: } 10.000-50.000 \text { hab. } \\
\text { tama3: } 50.000-500.000 \text { hab. } \\
\text { tama4: }>500.000 \text { hab. }\end{array}$ & Tama1 \\
\hline Renta & $\begin{array}{l}\text { Renta1: ocupado sin estudios. } \\
\text { Renta2: ocupado, estudios primarios. } \\
\text { Renta3: ocupado, estudios secundarios. } \\
\text { Renta4: ocupado, fp superior. } \\
\text { Renta5: ocupado, universitario. } \\
\text { Renta6: no ocupado. }\end{array}$ & Renta6 \\
\hline Edad & $\begin{array}{l}\text { edad1: } 0 \text { - } 14 \text { años. } \\
\text { edad2: } 15 \text { - } 29 \text { años. } \\
\text { edad3: } 30 \text { - } 39 \text { años. } \\
\text { edad4: } 40 \text { - } 49 \text { años. } \\
\text { edad5: } 50 \text { - } 64 \text { años. } \\
\text { edad6: } 65 \text { y más. }\end{array}$ & Edad6 \\
\hline Motivo & $\begin{array}{l}\text { Moti1: Ocio. } \\
\text { Moti2: Vacaciones. } \\
\text { Moti3: Profesionales. } \\
\text { Moti4: Visitar a familiares y amigos. } \\
\text { Moti5: Ir a segunda vivienda. }\end{array}$ & Moti5 \\
\hline Duración & Número de noches. & \\
\hline $\begin{array}{l}\text { CCAA } \\
\text { uniprovincial }\end{array}$ & $\begin{array}{l}=1 \text { si es uniprovincial. } \\
=0 \text { en otro caso. }\end{array}$ & No uniprovincial \\
\hline
\end{tabular}


TABLA 1.-Variables utilizadas y categoría de referencia (cont.)

\begin{tabular}{|c|c|c|}
\hline VARIABLES & CODIFICACIÓN & $\begin{array}{c}\text { CATEGORÍA } \\
\text { DE } \\
\text { REFERENCIA } \\
\end{array}$ \\
\hline Islas & $\begin{array}{l}=1 \text { si es comunidad insular. } \\
=0 \text { en otro caso. }\end{array}$ & No insular \\
\hline $\begin{array}{l}\text { Ciudades } \\
\text { autónomas }\end{array}$ & $\begin{array}{l}=1 \text { si es Ceuta y Melilla. } \\
=0 \text { en otro caso. }\end{array}$ & $\begin{array}{l}\text { No Ceuta y } \\
\text { Melilla }\end{array}$ \\
\hline $\begin{array}{l}\text { Provincia } \\
\text { limítrofe con } \\
\text { Francia }\end{array}$ & $\begin{array}{l}=1 \text { limítrofe con Francia. } \\
=0 \text { en otro caso. }\end{array}$ & No limítrofe \\
\hline $\begin{array}{l}\text { Provincia } \\
\text { limítrofe con } \\
\text { Portugal }\end{array}$ & $\begin{array}{l}=1 \text { limítrofe con Portugal. } \\
=0 \text { en otro caso. }\end{array}$ & No limítrofe \\
\hline $\begin{array}{l}\text { Dummies para } \\
\text { cada CCAA: } \\
\text { r1...r17 }\end{array}$ & $\begin{array}{l}=1 \text { residente en la CCAA. } \\
=0 \text { en otro caso. } \\
\text { (la correspondencia de los números de las variables } \\
\text { dummies con la CCAA se hace siguiendo la tabla que } \\
\text { acompaña al Gráfico 2) }\end{array}$ & $\begin{array}{l}\text { r7 (Castilla y } \\
\text { León) }\end{array}$ \\
\hline
\end{tabular}

\subsection{Resultados}

En las Tablas 2 y 3 se presentan los resultados de los dos modelos estimados. En ambos casos lo que se presentan son los coeficientes. Puesto que nuestra categoría de referencia son los viajes a la provincia de residencia, la probabilidad de elegir, por ejemplo, una provincia limítrofe de la misma comunidad (PLMC) vendrá dada por:

$\log \left(P(\right.$ tipodesino $=P L M C) / P($ tipodestino $=M P M C)=c_{P L M C}+\beta_{P L M C} *_{z_{i P L M C}}$

Tendremos una ecuación similar para el logaritmo del ratio de elegir cada una del resto de alternativas frente a la categoría de referencia. Por lo tanto, los coeficientes con un signo positivo implican una mayor preferencia por el tipo de destino correspondiente frente a viajar a la provincia de residencia.

En primer lugar vemos cómo el tamaño de los municipios de residencia es significativo y con coeficiente positivo en la mayoría de los casos (excepto para los viajes a una provincia limítrofe de una CCAA diferente a la de residencia de los residentes en municipios de 10.000 a 50.000 habitantes), esto es congruente con la hipótesis de que 
los residentes en municipios de mayor tamaño tienen una mayor probabilidad de viajar a un destino fuera de su provincia de residencia.

En lo que se refiere a la proxy de renta, vemos cómo en todos los casos los individuos con un nivel de renta mayor, tienen una mayor probabilidad de viajar fuera de su provincia de residencia. En el resto de los casos, los resultados no son claros. Aunque los viajes por motivos profesionales únicamente representan un $10 \%$ de la muestra, sería necesario comprobar cómo varía este efecto al eliminar este tipo de viajes de la muestra.

TABLA 2-.Resultados sin incluir dummies para cada CCAA

$\mathrm{N}=34052$

LR chi $2(115)=15261,27$

Prob $>$ chi $2=0.0000$

Pseudo R2 $=0.1412$

Log likelihood $=-46394,131$

\begin{tabular}{|c|c|c|c|c|c|c|c|c|c|c|}
\hline & \multicolumn{2}{|c|}{ PL MC } & \multicolumn{2}{|c|}{ PL OC } & \multicolumn{2}{|c|}{ OP MC } & \multicolumn{2}{|c|}{ OP OC } & \multicolumn{2}{|c|}{ EX } \\
\hline & Coef. & $P>|z|$ & Coef. & $P>|z|$ & Coef. & $\mathrm{P}>|\mathrm{z}|$ & Coef. & $P>|z|$ & Coef. & $P>|z|$ \\
\hline \multicolumn{11}{|l|}{ tama1 } \\
\hline tama2 & 0,147 & 0,000 & $-0,289$ & 0,000 & 0,086 & 0,320 & 0,161 & 0,000 & 0,095 & 0,236 \\
\hline tama3 & 0,414 & 0,000 & 0,190 & 0,000 & 0,459 & 0,000 & 0,356 & 0,000 & 0,248 & 0,001 \\
\hline tama4 & 1,237 & 0,000 & 0,181 & 0,036 & 0,097 & 0,596 & 0,731 & 0,000 & 1,061 & 0,000 \\
\hline renta1 & 0,042 & 0,772 & $-\mathbf{0 , 3 2 0}$ & 0,081 & $-0,490$ & 0,167 & $-0,218$ & 0,193 & $-0,031$ & 0,915 \\
\hline renta2 & $-0,038$ & 0,500 & 0,012 & 0,846 & $-0,045$ & 0,693 & 0,142 & 0,017 & $-0,141$ & 0,194 \\
\hline renta3 & 0,153 & 0,003 & 0,120 & 0,036 & 0,183 & 0,060 & 0,277 & 0,000 & 0,176 & 0,054 \\
\hline renta4 & 0,079 & 0,302 & 0,008 & 0,924 & $-0,349$ & 0,040 & 0,162 & 0,044 & $-0,052$ & 0,710 \\
\hline renta5 & 0,344 & 0,000 & 0,203 & 0,001 & 0,301 & 0,004 & 0,463 & 0,000 & 0,445 & 0,000 \\
\hline \multicolumn{11}{|l|}{ renta6 } \\
\hline edad1 & $\mathbf{0 , 1 3 0}$ & 0,054 & 0,122 & 0,102 & 0,122 & 0,393 & $-0,157$ & 0,028 & 0,405 & 0,003 \\
\hline edad2 & 0,275 & 0,000 & $\mathbf{0 , 1 2 9}$ & 0,094 & 0,486 & 0,000 & 0,093 & 0,187 & 0,590 & 0,000 \\
\hline edad3 & 0,287 & 0,000 & 0,240 & 0,004 & 0,340 & 0,028 & 0,165 & 0,034 & 0,953 & 0,000 \\
\hline edad4 & 0,304 & 0,000 & 0,322 & 0,000 & 0,393 & 0,010 & 0,211 & 0,006 & 0,872 & 0,000 \\
\hline edad5 & 0,270 & 0,000 & 0,274 & 0,000 & 0,307 & 0,036 & 0,326 & 0,000 & 0,882 & 0,000 \\
\hline
\end{tabular}


TABLA 2-.Resultados sin incluir dummies para cada CCAA (cont.)

\begin{tabular}{|c|c|c|c|c|c|c|c|c|c|c|}
\hline & \multicolumn{2}{|c|}{ PL MC } & \multicolumn{2}{|c|}{ PL OC } & \multicolumn{2}{|c|}{ OP MC } & \multicolumn{2}{|c|}{ OP OC } & \multicolumn{2}{|c|}{ EX } \\
\hline & Coef. & $\mathrm{P}>|\mathrm{z}|$ & Coef. & $P>|z|$ & Coef. & $\mathrm{P}>|\mathrm{z}|$ & Coef. & $\mathrm{P}>|\mathrm{z}|$ & Coef. & $\mathrm{P}>|\mathrm{z}|$ \\
\hline moti1 & 1,214 & 0,000 & 1,251 & 0,000 & 2,235 & 0,000 & 3,118 & 0,000 & 4,399 & 0,000 \\
\hline moti2 & 0,811 & 0,000 & 0,836 & 0,000 & 1,118 & 0,000 & 1,045 & 0,000 & 2,110 & 0,000 \\
\hline moti3 & 0,672 & 0,000 & 0,397 & 0,000 & 1,194 & 0,000 & 1,264 & 0,000 & 1,759 & 0,000 \\
\hline moti4 & 0,507 & 0,000 & 0,627 & 0,000 & 0,740 & 0,000 & 0,759 & 0,000 & 0,640 & 0,000 \\
\hline \multicolumn{11}{|l|}{ moti5 } \\
\hline duracion & $-0,063$ & 0,000 & $-0,021$ & 0,000 & 0,002 & 0,829 & 0,029 & 0,000 & 0,046 & 0,000 \\
\hline cuniprov & $-35,256$ & 1,000 & 0,530 & 0,000 & $-35,218$ & 1,000 & 0,211 & 0,000 & $-0,160$ & 0,050 \\
\hline islas & $-35,641$ & 1,000 & $-36,274$ & 1,000 & $-0,265$ & 0,032 & $-1,620$ & 0,000 & $-0,790$ & 0,000 \\
\hline ceu_meli & $-3,213$ & 1,000 & $-38,929$ & 1,000 & $-3,551$ & 1,000 & 6,115 & 0,000 & 8,165 & 0,000 \\
\hline lim_Fr & 0,700 & $\mathbf{0 , 0 0 0}$ & $-0,074$ & 0,218 & $-0,920$ & $\mathbf{0 , 0 0 0}$ & $\mathbf{0 , 2 9 7}$ & 0,000 & 1,199 & 0,000 \\
\hline lim_Por & $-0,022$ & 0,602 & $-1,264$ & 0,000 & $-0,337$ & 0,000 & $-0,124$ & 0,015 & 0,749 & 0,000 \\
\hline _cons & $-1,050$ & 0,000 & $-1,381$ & 0,000 & $-3,285$ & 0,000 & $-1,944$ & 0,000 & $-5,216$ & 0,000 \\
\hline
\end{tabular}

Respecto a la edad, se puede decir en primer lugar que los individuos de 30 a 64 años tienen una mayor probabilidad de viajar a cualquier lugar fuera de su provincia de residencia que los mayores de 65 (cuyos viajes son en mayor proporción a la provincia de residencia). En segundo lugar, podemos decir que los individuos entre 15 y 29 años, tienen una probabilidad mayor de elegir como destino un lugar fuera de la provincia de residencia, excepto una provincia no limítrofe de otra CCAA.

Se comprueba cómo para viajes más largos, existe una menor probabilidad de que el destino elegido sea una provincia limítrofe frente a los viajes intra-provinciales. Por el contrario, una mayor duración del viaje aumentará la preferencia por los viajes a provincias no limítrofes de otras CCAA o al extranjero. De tal forma que un incremento unitario en la duración del viaje, reducirá el logaritmo del ratio de probabilidad de elegir una provincia limítrofe en -0.063 para provincias de la CCAA de residencia y en un -0.021 para provincias de otra CCAA, mientras que aumenta en 0.029 y 0.046 el logaritmo del ratio de probabilidad entre los viajes a provincias no limítrofes de otras CCAA y del extranjero con los viajes a la provincia de residencia, respectivamente.

Sobre el motivo del viaje, podemos decir que existen diferencias significativas en todos los casos entre la probabilidad de elegir como destino la provincia de residencia o cualquier otro destino según el motivo del viaje. La probabilidad de viajar al extranjero 
aumenta para los viajes de ocio (4.399), vacaciones (2.110) o incluso profesionales (1.759). Sin embargo, en los viajes para visitar a familiares y amigos la diferencia no es tan grande (0.640).

En relación a las dummies sobre las características geográficas de algunas comunidades, podemos señalar en primer lugar, que el hecho de ser una comunidad uniprovinicial aumenta la probabilidad de realizar viajes a otra comunidad $(0.53$ para provincias limítrofes y 0.211 para no limítrofes). El resultado anterior es lógico dado el pequeño tamaño de las comunidades uniprovinciales. Por otro lado, reduce la probabilidad de elegir un destino en el extranjero (-0.160). Para las islas, sin embargo, encontramos que se reduce la probabilidad de que el destino elegido sean otras comunidades autónomas (-1.620) o incluso el extranjero. Por el contrario, los residentes en Ceuta y Melilla elegirán con una mayor probabilidad de otra CCAA (6.115) y el extranjero (8.165), tal y como veíamos en el Gráfico 2.

El hecho de ser residentes en una de las provincias limítrofes con Portugal, reduce la probabilidad de elegir otras provincias como destino, mientras que con mayor probabilidad realizarán viajes al extranjero (0.749). Este resultado tiene que ver con la fácil accesibilidad hacia Portugal, en contraposición con su situación geográfica en España. Sin embargo, los residentes en provincias limítrofes con Francia presentan una mayor preferencia por un destino extranjero, pero también por una provincia limítrofe de su CCAA de residencia o por una provincia no limítrofe de otra comunidad.

En la Tabla 3, presentamos los coeficientes estimados de la especificación en la que hemos incluido dummies específicas para cada comunidad y sus p-valores asociados. Vemos que el poder explicativo del modelo ha aumentado (el pseudo $\mathrm{R}^{2}$ de $\mathrm{Mc}$ Fadden ha pasado de un $14 \%$ a un $19 \%$ ), por lo que podemos decir que controlar la heterogeneidad presente en las comunidades autónomas nos aporta información adicional sobre los destinos de los viajes, debido a que existen diferencias significativas entre ellas. Los coeficientes estimados para el resto de las variables no se han modificado demasiado, aunque en algunos casos han perdido significatividad.

Respecto a las dummies referentes a las comunidades autónomas podemos decir, que por un lado, los residentes en las comunidades insulares tienen una probabilidad menor de viajar a una provincia de otra comunidad diferente a la de residencia, debido a los mayores costes de transporte que les supone a los residentes en esas comunidades viajar a otras comunidades. Los residentes en Baleares tienen una mayor probabilidad de viajar al extranjero (0.580), al contrario que los residentes en Canarias (-1.296). Este diferente comportamiento entre los residentes de ambas comunidades se veía en el Gráfico 2, en el que Canarias aparecía con una mayor tendencia a viajar dentro de su propia comunidad que los residentes en Baleares. A partir de los resultados obtenidos 
podemos decir que los residentes en ambas comunidades tienen menos probabilidad de viajar a otras comunidades que a su propia provincia, sin embargo mientras que los residentes en Baleares eligen con mayor probabilidad el extranjero como destino de sus viajes, los residentes en Canarias presentan muestran una mayor preferencia por su provincia de residencia. Este diferente comportamiento de Baleares y Canarias hacia el exterior, no era captado al introducir una dummy para ambas en lugar de una para cada una de ellas.

TABLA 3.- Resultados incluyendo dummies para cada CCAA

$\mathrm{N}=34.052$

LR chi 2(185) $=21.239,29$

Prob $>$ chi $2=0$

Pseudo R2 = 0,1966

Log likelihood $=-43.405,121$

\begin{tabular}{|c|c|c|c|c|c|c|c|c|c|c|}
\hline & \multicolumn{2}{|c|}{ PL MC } & \multicolumn{2}{|c|}{ PL OC } & \multicolumn{2}{|c|}{ OP MC } & \multicolumn{2}{|c|}{ OP OC } & \multicolumn{2}{|c|}{ EX } \\
\hline & Coef. & $\mathrm{P}>|\mathrm{z}|$ & Coef. & $\mathrm{P}>|\mathrm{z}|$ & Coef. & $\mathrm{P}>|\mathrm{z}|$ & Coef. & $\mathrm{P}>|\mathrm{z}|$ & Coef. & $\mathrm{P}>|\mathrm{z}|$ \\
\hline \multicolumn{11}{|l|}{ tama1 } \\
\hline tama2 & 0,105 & 0,015 & $-0,066$ & 0,195 & 0,073 & 0,415 & 0,283 & 0,000 & 0,098 & 0,232 \\
\hline tama3 & 0,370 & 0,000 & 0,307 & 0,000 & 0,343 & 0,000 & 0,403 & 0,000 & 0,217 & 0,005 \\
\hline tama4 & 0,932 & $\mathbf{0 , 0 0 0}$ & 0,684 & $\mathbf{0 , 0 0 0}$ & $-0,052$ & 0,784 & 0,889 & 0,000 & 0,941 & 0,000 \\
\hline renta1 & 0,120 & 0,416 & $-0,277$ & 0,145 & $-0,462$ & 0,195 & $-0,143$ & 0,408 & 0,009 & 0,974 \\
\hline renta2 & $-0,014$ & 0,802 & 0,002 & 0,972 & $-0,033$ & 0,777 & 0,131 & $\mathbf{0 , 0 3 3}$ & $-0,167$ & 0,130 \\
\hline renta3 & 0,111 & $\mathbf{0 , 0 3 2}$ & 0,140 & 0,018 & 0,225 & 0,022 & 0,256 & $\mathbf{0 , 0 0 0}$ & 0,103 & 0,268 \\
\hline renta4 & $-0,030$ & 0,700 & 0,016 & 0,860 & $-0,323$ & 0,060 & 0,076 & 0,366 & $-0,199$ & 0,162 \\
\hline renta5 & 0,338 & 0,000 & 0,147 & 0,024 & 0,356 & 0,001 & 0,402 & 0,000 & 0,405 & 0,000 \\
\hline \multicolumn{11}{|l|}{ renta6 } \\
\hline edad1 & 0,114 & 0,097 & 0,166 & $\mathbf{0 , 0 3 3}$ & 0,091 & 0,528 & $-0,125$ & $\mathbf{0 , 0 8 9}$ & 0,440 & 0,002 \\
\hline edad2 & 0,306 & 0,000 & 0,158 & 0,048 & 0,425 & 0,002 & 0,156 & 0,032 & 0,668 & 0,000 \\
\hline edad3 & 0,310 & 0,000 & 0,320 & 0,000 & 0,300 & 0,056 & 0,271 & 0,001 & 1,069 & 0,000 \\
\hline edad4 & 0,365 & 0,000 & 0,320 & 0,000 & 0,359 & 0,019 & 0,288 & 0,000 & 0,972 & 0,000 \\
\hline edad5 & 0,278 & 0,000 & 0,310 & 0,000 & 0,307 & 0,038 & 0,370 & 0,000 & 0,937 & 0,000 \\
\hline \multicolumn{11}{|l|}{ edad6 } \\
\hline moti1 & 1,235 & 0,000 & 1,419 & 0,000 & 2,169 & 0,000 & 3,270 & 0,000 & 4,496 & 0,000 \\
\hline moti2 & 0,776 & 0,000 & 0,910 & 0,000 & 1,071 & 0,000 & 1,101 & 0,000 & 2,130 & 0,000 \\
\hline
\end{tabular}


TABLA 3.-Resultados incluyendo dummies para cada CCAA (cont.)

\begin{tabular}{|c|c|c|c|c|c|c|c|c|c|c|}
\hline moti3 & 0,657 & 0,000 & 0,475 & 0,000 & 1,147 & 0,000 & 1,332 & 0,000 & 1,786 & 0,000 \\
\hline \multirow[t]{3}{*}{ moti4 } & 0,484 & 0,000 & 0,678 & 0,000 & 0,717 & 0,000 & 0,793 & 0,000 & 0,649 & 0,000 \\
\hline & \multicolumn{2}{|c|}{ PL MC } & \multicolumn{2}{|c|}{ PL OC } & \multicolumn{2}{|c|}{ OP MC } & \multicolumn{2}{|c|}{ OP OC } & \multicolumn{2}{|c|}{ EX } \\
\hline & Coef. & $\mathrm{P}>|\mathrm{z}|$ & Coef. & $\mathrm{P}>|\mathrm{z}|$ & Coef. & $\mathrm{P}>|\mathrm{z}|$ & Coef. & $\mathrm{P}>|\mathrm{z}|$ & Coef. & $\mathrm{P}>|\mathrm{z}|$ \\
\hline \multicolumn{11}{|l|}{ moti5 } \\
\hline duracion & $-0,067$ & $\mathbf{0 , 0 0 0}$ & $-0,021$ & 0,000 & $-0,002$ & 0,798 & 0,028 & $\mathbf{0 , 0 0 0}$ & 0,044 & 0,000 \\
\hline $\mathrm{r} 1$ & 0,246 & 0,000 & $-2,377$ & 0,000 & 0,309 & $\mathbf{0 , 0 0 0}$ & $-1,292$ & 0,000 & $-0,158$ & 0,190 \\
\hline $\mathrm{r} 2$ & 0,136 & 0,074 & $-0,245$ & 0,006 & $-1,765$ & $\mathbf{0 , 0 0 0}$ & $-0,409$ & 0,000 & 0,013 & 0,938 \\
\hline $\mathrm{r} 3$ & 4,602 & 1,000 & $-0,872$ & 0,000 & $-35,160$ & 1,000 & $-1,298$ & 0,000 & $-1,097$ & 0,000 \\
\hline $\mathrm{r} 4$ & $-34,676$ & 1,000 & $-35,095$ & 1,000 & $-35,349$ & 1,000 & $-0,875$ & 0,000 & 0,580 & 0,006 \\
\hline r5 & 35,374 & 1,000 & $-35,807$ & 1,000 & $-0,612$ & 0,000 & $-2,631$ & 0,000 & $-1,296$ & 0,000 \\
\hline r6 & $-34,162$ & 1,000 & 0,429 & 0,000 & $-34,644$ & 1,000 & $-0,142$ & 0,228 & 0,261 & 0,298 \\
\hline \multicolumn{11}{|l|}{ r7 } \\
\hline r8 & $-0,705$ & 0,000 & 0,506 & 0,000 & $-0,862$ & $\mathbf{0 , 0 0 0}$ & $-0,114$ & $\mathbf{0 , 0 8 8}$ & 0,053 & 0,739 \\
\hline r9 & 0,828 & 0,000 & -1 & 0,000 & $-1,196$ & 0,000 & 20 & 00 & 1,090 & 0,000 \\
\hline r10 & $-0,248$ & 0,001 & $-0,517$ & 0,000 & $-1,266$ & 0,000 & $-0,310$ & 0,000 & 0,370 & 0,018 \\
\hline $\mathrm{r} 11$ & $-1,026$ & $\mathbf{0 , 0 0 0}$ & 0,864 & 0,000 & $-34,378$ & 1,000 & $-0,574$ & 0,000 & $-1,202$ & 0,000 \\
\hline $\mathrm{r} 12$ & 0,326 & 0,000 & $-1,619$ & 0,000 & $-0,092$ & 0,421 & $-0,893$ & 0,000 & 0,246 & 0,053 \\
\hline r13 & 33,775 & 1,000 & 0,441 & $\mathbf{0 , 0 0 0}$ & $-34,024$ & 1,000 & $\mathbf{0 , 5 4 5}$ & $\mathbf{0 , 0 0 0}$ & 0,889 & 0,000 \\
\hline r14 & $-34,439$ & 1,000 & $-0,061$ & 0,559 & $-34,974$ & 1,000 & $-0,933$ & 0,000 & $-0,400$ & 0,127 \\
\hline r15 & $-34,329$ & 1,000 & 0,699 & 0,000 & $-35,027$ & 1,000 & $-0,375$ & 0,004 & 0,151 & 0,468 \\
\hline $\mathrm{r} 16$ & $\mathbf{0 , 9 5 7}$ & 0,000 & 1,422 & 0,000 & $-33,275$ & 1,000 & 1,528 & 0,000 & 1,954 & 0,000 \\
\hline $\mathrm{r} 17$ & $-33,643$ & 1,000 & 0,454 & $\mathbf{0 , 0 0 0}$ & $-34,190$ & 1,000 & $\mathbf{0 , 8 8 7}$ & $\mathbf{0 , 0 0 0}$ & $\mathbf{0 , 8 0 8}$ & 0,002 \\
\hline ceu_meli & $-37,292$ & 1,000 & $-37,685$ & 1,000 & $-37,881$ & 1,000 & 6,012 & 0,000 & 8,319 & 0,000 \\
\hline $\operatorname{limfr}$ & 0,233 & 0,001 & $-0,474$ & 0,000 & 0,440 & 0,075 & 0,114 & 0,172 & 0,694 & 0,000 \\
\hline limpor & 0,222 & 0,000 & $-1,788$ & 0,000 & $-0,107$ & 0,288 & 0,056 & 0,418 & 1,244 & 0,000 \\
\hline _cons & $-1,119$ & 0,000 & $-1,228$ & 0,000 & $-2,954$ & 0,000 & $-1,739$ & 0,000 & $-5,531$ & 0,000 \\
\hline
\end{tabular}


Los residentes en las comunidades uniprovinciales, cuando se intentaba controlar esta característica con una única dummy (cuniprov), presentaban una mayor probabilidad de viajar a otras CCAA que a su propia comunidad, frente a una menor probabilidad de viajar al extranjero. Sin embargo, ahora vemos cómo el comportamiento varía según la CCAA de residencia de tal forma que encontramos:

- CCAA con mayores probabilidades de realizar tanto un viaje a otra CCAA, limítrofe o no, como al extranjero. En este grupo están La Rioja o Madrid.

- CCAA que tienen una menor probabilidad de realizar tanto un viaje a otra CCAA, limítrofe o no, o al extranjero. En este grupo están Asturias o Murcia (en este caso los coeficientes de Murcia, para los viajes a provincias limítrofes o al extranjero no son significativos).

- CCAA con una mayor probabilidad de realizar un viaje a una CCAA limítrofe y una menor probabilidad a otras CCAA no limítrofes. Este es el caso de Navarra.

- CCAA con una mayor probabilidad de realizar viajes a CCAA no limítrofes y una menor probabilidad de realizar un viaje a una CCAA no limítrofe. Este es el caso de Cantabria.

Respecto a los residentes en el resto de las CCAA destaca el caso de los residentes en Andalucía, cuyo tamaño y variada oferta turística, favorece que se obtenga una mayor probabilidad de elegir un destino dentro de la propia comunidad ( 0.246 para provincias limítrofes y 0.309 para no limítrofes), y una menor probabilidad de viajar a otra (-2.377 para provincias limítrofes y -1.292 para no limítrofes). De hecho, es la única comunidad que presenta una mayor probabilidad de elegir una provincia no limítrofe de la misma comunidad.

Destaca también el caso de los residentes en Cataluña, Aragón y Galicia, con una mayor probabilidad de elegir una provincia limítrofe dentro de la misma CCAA, pero menores probabilidades para el resto de los casos (excepto los destinos internacionales). Por el contrario, los residentes en el País Vasco eligen con una probabilidad mayor de elegir como destino una comunidad diferente a la de residencia, incluso aunque no sea limítrofe que elegir la provincia en la que residen.

Por último, los residentes en las provincias que pertenecen a las comunidades de Castilla La Mancha, comunidad Valenciana y Extremadura tienen una menor probabilidad de elegir como destino de sus viajes otras provincias de la CCAA de residencia o de otra, excepto en el caso de Extremadura, que presenta mayor probabilidad de elegir una provincia limítrofe de otra comunidad. 


\section{CONCLUSIONES}

A través de este artículo hemos tratado de comprobar cómo las características particulares de las provincias de residencia de los individuos, ya sean geográficas, de accesibilidad, culturales, etc. afectan a la elección de destino de los viajes de los individuos. Por lo tanto, el objetivo es determinar en qué medida existe una cierta heterogeneidad regional que debemos controlar a la hora de realizar nuestros análisis.

En primer lugar hemos elaborado un análisis descriptivo de los principales factores determinantes de elegir un tipo de destino determinado que se encuentran en la literatura. A través de este análisis hemos confirmado que los habitantes de los municipios de mayor tamaño realizan una mayor proporción de viajes a provincias limítrofes de su CCAA de residencia, incluso en mayor medida que a su propia provincia. Por otro lado, se verifica que los ocupados viajan en mayor proporción a otras comunidades, mientras que los jubilados elegirán con mayor probabilidad su propia provincia como destino. Se ha constatado también que un nivel de estudios superior aumenta la probabilidad de viajar al extranjero. Hemos verificado el importante efecto que las motivaciones para realizar el viaje tiene sobre el destino elegido en el mismo sentido en el que Nicolau y Más (2006) encontraban un efecto moderador sobre la distancia y el precio.

Respecto a la heterogeneidad regional, encontramos por un lado un grupo de comunidades que realizan una gran cantidad de viajes a sus propias comunidades, en algunos casos por su situación geográfica como es el caso de Canarias, en otras por una cuestión cultural como es el caso de Galicia o Cataluña. El caso de Cataluña es contrario a los resultados obtenidos en (Llano y de la Mata, 2009). También es de destacar el comportamiento de los residentes en el País Vasco, siendo una comunidad autónoma fronteriza con Francia, con lengua y características culturales propias, es la única comunidad no uniprovincial (junto con Castilla La Mancha) que se encuentra dentro del grupo de las que viajan en mayor proporción a otras CCAA que el grupo que representa el comportamiento medio. Destaca el comportamiento viajero extraordinariamente ligado a los viajes al extranjero de Ceuta y Melilla.

Partiendo de estos resultados, existen algunas extensiones necesarias del trabajo para poder sacar conclusiones de manera más firme. En primer lugar, es necesario desarrollar un análisis diferente según el motivo de los viajes. En segundo lugar, para evitar el sesgo que se introduce al tener únicamente en cuenta la información de los individuos que sí efectúan viajes, podemos realizar un análisis con el objetivo de cotejar en qué medida la toma de decisiones se llevan a cabo de forma jerárquica haciendo uso de un logit anidado. Adicionalmente, podemos analizar cuales son los determinantes 
de la duración de la estancia o de la distancia que un individuo es capaz de recorrer a la hora de realizar sus viajes. En tercer lugar y con el objetivo de ver en qué medida los resultados obtenidos son consistentes, es necesario elaborar un nuevo análisis en el que se comparen Movilia 2001 con Movilia 2007 o con otras fuentes como las encuestas del Instituto de Estudios Turísticos. Por último, es necesario aplicar procedimientos que nos permitan corregir los efectos que el número de provincias limítrofes o el número de provincias de cada CCAA puedan tener sobre los resultados.

\section{REFERENCIAS BIBLIOGRÁFICAS}

Alegre, J. y Pou, L. (2004) «Microeconomic determinants of the probability of tourism consumption», Tourism Economics, 10 (2) 2004: 125-144.

- (2006) «The length of stay in the demand for tourism», Tourism Management, 27 (6) 2006: 1343-1355.

Artal-Tur, A.; García-Sánchez, A.; Navaro-Azorín, J. M. (2010), «The length of stay for sun and sand tourism and the roel of holiday characteristics» (Work in progress).

Aznar Marquez, J. y Nicolini, R. (2007) «El sector turístico en la Comunidad Valenciana: Unos elementos de análisis de la demanda en el marco de la economía geográfica», Revista de Estudios Regionales, 79 (2007): 43-72.

Baltas, G. (2006), «Econometric Models for Discrete Choice Analysis of Travel and Tourism Demand», Journal of Travel and Tourism Marketing, 21 (4) 2006: 25-40.

Basala, S y Klenosky, D. (2001) «Travel style preferences for visiting a novel destination: A conjoint investigation across the novelty-familiarity continuum», Journal of Travel Research 40 (2) 2001: 172-183.

BECKER, G. S. (1965) «A theory of the allocation of time», The Economic Journal, 75 (299) 1965: 493-517.

CAI, LiPING A. (1998) «Analyzing household food expenditure patterns on trips and vacations: a tobit model», Journal of Hospitality and Tourism Research, 22 (4) 1998: 338-358.

CAÑAdA, A. (2002) «El turismo interior en España y su dimensión espacial: las regiones como exportadoras e importadoras de turismo interior», Cuadernos de Información Económica, 169: $137-140$.

Collins, D. y Tisdell, C. (2002) «Age-related lifecycles. Purpose variations», Annals of Tourism Research, 29 (3) 2002: 801-818.

DARDis, R.; Derrick, F.; LeHFeld, A. y Wolfe, K. E. (1981) «Cross section studies of recreation expenditures in the United States» Journal of Leisure Research, 13 (3) 1981: 181-194. 
Dardis, R.; Soberon-Ferrer, H. y Patro, D. (1994) «Analysis of leisure expenditures in the United States», Journal of Leisure Research, 13 (3) 1994: 309-321.

Deaton, A. (1997) The Analysis of Household Surveys: A Microeconometric Approach to Development Policy, World Bank and John Hopkins University Press, Baltimore, MD.

Eymann, A.; Ronning, G. (1997) «Microeconometric models of tourists' destination choice», Regional Science and Urban Economics, 27 (1997): 735-761.

Ghez, G. y Becker, G. (1975) «The Allocation of Time and Goods over the Life Cycle «, NBER.

Henderson, J. C. (2003) «Managing tourism and Islam in Peninsular Malaysia», Tourism Management 24 (2003): 447-456.

Imm N. S.; Anne Lee, S.; Soutar, G. N. (2007): «Tourists' intention to visit a country: The impact of cultural distance», Tourism Management, 28 (2007): 1497-1506.

INE (2004) «Cuenta Satélite del Turismo en España: Nota metodológica». Subdirección General de Cuentas Nacionales (2004).

LANCASTER, K. J. (1966). «A new approach to consumer theory», Journal of Political Economy, 74 (2) 1966: 132-157.

LARGo JimÉneZ, J. (1976) «El turismo y el mercado interior en España», Estudios turísticos, 49-50: 71-99

LAWsON, R. W. (1991) «Patterns of tourist expenditure and types of vacation across the family life cycle», Journal of Travel Research, 29 (4) 1991: 12-18.

Lim, C. (1999) «A meta-analytical review of international Tourism Demand», Journal of Travel Research, 37 (1999): 273-284.

Llano, C. y DE LA Mata, T. (2009) «El comercio interregional en España: Una estimación de los flujos bilaterales del sector Turismo» Información Comercial Española, 848 (2009): 67-88.

Manski, C. F. (1977) «The Structure of Random Utility Models», Theory and Decision, 8 (1977): 229-254.

Martinez-Garcia, E. y Raya, J. M. (2008) «Length of stay for low-cost tourism», Tourism Management, 29 (6) 2008: 1064-1075.

Mc Fadden, D. (1974) «Conditional Logit Analysis of Qualitative Choice Behaviour», Frontiers in Econometrics, eds. Zarembka, Nueva York: Academic Press, 1974.

Ministerio de Fomento (2007). Encuesta de Movilidad de las Personas Residentes en España (MOVILIA).

http://www.fomento.es/MFOM/LANG_CASTELLANO/INFORMACION_MFOM/ INFORMACION_ESTADISTICA/Movilidad/Movilia2006_2007/

Millán Escriche, M. (2004) «Turismo en la Región de Murcia: evolución de la oferta turística de interior y su distribución espacial», Cuadernos de turismo, 13 (2004): 51-72. 
Nicolau, J. L.; Más, F. J. (2005) «Stochastic modeling. A three-stage tourist choice process», Annals of Tourism Research, 32 (1) 2005: 49-69.

- (2006) «The influence of distance and prices on the choice of tourist destinations: The moderating role of motivations», Tourism Management, 27 (2006): 982-996.

Nicolau, J. L.; Más, F. J. (2008) «Sequential choice behavior: Going on vacation and type of destination» Tourism Management, 29 (2008): 1023-1034.

Opperman, M. (1995) «Family Life Cycle and Cohort Effects: A Study of Travel Patterns of German Residents», Journal of Travel and Tourism Marketing, 4 (1) 1995: 23-44.

Riera, A. (2000) «Modelos de elección discreta y coste del viaje. Los espacios naturales protegidos en Mallorca», Revista de Economía Aplicada, 24 (8) 2000: 181-201.

Simon, H. A. (1955) «A behavioural model of rational choice», Journal of Economics, 69 (1955): 99-118.

SoNG, H. y LI, G. (2008) «Tourism demand modelling and forecasting-A review of recent research», Tourism Management, 29 (2) 2008: 203-220.

Thurstone L. L. (1927) «A Law of Comparative Judgement», Psychological Review, 34 (1927): 273-286.

- (1945) «The prediction of choice», Psychometrika, 10 (4) 1945: 237-253.

UsACH Domingo, J. (1998): «Análisis de los flujos interregionales de la demanda turística interna española», Estudios turísticos, 136 (1998): 27-44.

Walsh, R.; Kun, John; M., J. y Hof, J. (1992) «Effect of Price on Forecasts of Participation in Fish and Wildlife Recreation: An Aggregate Demand Model», Journal of Leisure Research, 24 (2) 1992: 140-156.

WitT, S. F.; WitT, C. A. (1995) «Forecasting tourism demand: A review of empirical research», International Journal of Forecasting, 11 (3) 1995: 447-475. 


\section{ANEXO}

TABLA 4.-Descripción de las variables

\begin{tabular}{|c|c|c|c|c|c|c|}
\hline Variable & Definición & Media & $\begin{array}{l}\text { Desv. } \\
\text { Típica }\end{array}$ & Min & $\operatorname{Max}$ & Obs \\
\hline \multicolumn{7}{|c|}{ Variable dependiente: tipo de destino } \\
\hline Tipodestino & $\begin{array}{l}1 \mathrm{MP} \text { MC; } 2 \text { PL MC; } 3 \text { PL } \\
\text { OC; } 4 \text { OP MC; } 5 \text { OP OC; } \\
6 \text { EX }\end{array}$ & 2,775 & 1,673 & 1 & 6 & 34.052 \\
\hline \multicolumn{7}{|c|}{ Tamaño del municipio de residencia } \\
\hline tama1 & $<10.000$ habitantes & 0,324 & 0,468 & 0 & 1 & 34.052 \\
\hline tama2 & 10.000 - 50.000 habitantes & 0,240 & 0,427 & 0 & 1 & 34.052 \\
\hline tama3 & $\begin{array}{l}50.000-500.000 \\
\text { habitantes }\end{array}$ & 0,377 & 0,485 & 0 & 1 & 34.052 \\
\hline tama4 & $>500.000$ habitantes & 0,059 & 0,236 & 0 & 1 & 34.052 \\
\hline \multicolumn{7}{|c|}{ Grupo de edad } \\
\hline edad1 & 0 - 14 años & 0,157 & 0,364 & 0 & 1 & 34.052 \\
\hline edad2 & $15-29$ años & 0,212 & 0,409 & 0 & 1 & 34.052 \\
\hline edad3 & 30 - 39 años & 0,168 & 0,374 & 0 & 1 & 34.052 \\
\hline edad4 & 40 - 49 años & 0,185 & 0,389 & 0 & 1 & 34.052 \\
\hline edad5 & 50 - 64 años & 0,192 & 0,394 & 0 & 1 & 34.052 \\
\hline edad6 & 65 y más & 0,085 & 0,280 & 0 & 1 & 34.052 \\
\hline \multicolumn{7}{|c|}{ Grupo de actividad } \\
\hline ocup1 & Ocupados & 0,522 & 0,500 & 0 & 1 & 34.052 \\
\hline ocup2 & Labores del hogar & 0,096 & 0,294 & 0 & 1 & 34.052 \\
\hline ocup3 & Parados y otros & 0,034 & 0,181 & 0 & 1 & 34.052 \\
\hline ocup4 & Jubilados & 0,108 & 0,310 & 0 & 1 & 34.052 \\
\hline ocup5 & Estudiantes & 0,220 & 0,414 & 0 & 1 & 34.052 \\
\hline ocup6 & Menor no escolarizado & 0,021 & 0,143 & 0 & 1 & 34.052 \\
\hline \multicolumn{7}{|c|}{ Nivel de estudios } \\
\hline estu1 & $\begin{array}{l}\text { Sin estudios o primaria sin } \\
\text { terminar }\end{array}$ & 0,175 & 0,380 & 0 & 1 & 34.052 \\
\hline estu2 & Primarios & 0,270 & 0,444 & 0 & 1 & 34.052 \\
\hline estu3 & Secundarios & 0,294 & 0,456 & 0 & 1 & 34.052 \\
\hline
\end{tabular}




\begin{tabular}{|c|c|c|c|c|c|c|}
\hline \multicolumn{7}{|c|}{ Nivel de estudios } \\
\hline estu4 & FP superior & 0,070 & 0,256 & 0 & 1 & 34.052 \\
\hline estu5 & Universitarios & 0,191 & 0,393 & 0 & 1 & 34.052 \\
\hline \multicolumn{7}{|c|}{ Proxy de renta } \\
\hline rental & $\begin{array}{l}\text { Ocupado*estu1 (renta más } \\
\text { baja) }\end{array}$ & 0,011 & 0,103 & 0 & 1 & 34.052 \\
\hline renta2 & Ocupado*estu2 & 0,120 & 0,325 & 0 & 1 & 34.052 \\
\hline renta3 & Ocupado*estu3 & 0,182 & 0,386 & 0 & 1 & 34.052 \\
\hline renta4 & Ocupado*estu4 & 0,053 & 0,224 & 0 & 1 & 34.052 \\
\hline renta5 & $\begin{array}{l}\text { Ocupado*estu5 (renta más } \\
\text { alta) }\end{array}$ & 0,156 & 0,363 & 0 & 1 & 34.052 \\
\hline \multicolumn{7}{|c|}{ Motivo del viaje } \\
\hline moti1 & Ocio & 0,135 & 0,341 & 0 & 1 & 34.052 \\
\hline moti2 & Vacaciones & 0,342 & 0,475 & 0 & 1 & 34.052 \\
\hline moti3 & Profesionales & 0,076 & 0,265 & 0 & 1 & 34.052 \\
\hline moti4 & $\begin{array}{l}\text { Visitar a familiares y } \\
\text { amigos }\end{array}$ & 0,190 & 0,392 & 0 & 1 & 34.052 \\
\hline moti5 & Ir a $2^{\mathrm{a}}$ residencia & 0,139 & 0,346 & 0 & 1 & 34.052 \\
\hline \multicolumn{7}{|c|}{ Comunidad autónoma de residencia } \\
\hline $\mathrm{r} 1$ & Andalucía $=1,0$ otro caso & 0,155 & 0,362 & 0 & 1 & 34.052 \\
\hline r2 & Aragón $=1,0$ otro caso & 0,063 & 0,243 & 0 & 1 & 34.052 \\
\hline $\mathrm{r} 3$ & Asturias $=1,0$ otro caso & 0,019 & 0,138 & 0 & 1 & 34.052 \\
\hline $\mathrm{r} 4$ & Baleares $=1,0$ otro caso & 0,011 & 0,106 & 0 & 1 & 34.052 \\
\hline $\mathrm{r} 5$ & Canarias $=1,0$ otro caso & 0,024 & 0,152 & 0 & 1 & 34.052 \\
\hline r6 & Cantabria $=1,0$ otro caso & 0,019 & 0,138 & 0 & 1 & 34.052 \\
\hline r7 & $\begin{array}{l}\text { Castilla y León }=1,0 \text { otro } \\
\text { caso }\end{array}$ & 0,179 & 0,383 & 0 & 1 & 34.052 \\
\hline r8 & $\begin{array}{l}\text { Castilla-La Mancha }=1,0 \\
\text { otro caso }\end{array}$ & 0,099 & 0,298 & 0 & 1 & 34.052 \\
\hline r9 & Cataluña $=1,0$ otro caso & 0,089 & 0,285 & 0 & 1 & 34.052 \\
\hline $\mathrm{r} 10$ & $\begin{array}{l}\text { Com. Valenciana }=1,0 \\
\text { otro caso }\end{array}$ & 0,058 & 0,234 & 0 & 1 & 34.052 \\
\hline r11 & $\begin{array}{l}\text { Extremadura }=1,0 \text { otro } \\
\text { caso }\end{array}$ & 0,039 & 0,195 & 0 & 1 & 34.052 \\
\hline $\mathrm{r} 12$ & Galicia $=1,0$ otro caso & 0,076 & 0,265 & 0 & 1 & 34.052 \\
\hline
\end{tabular}




\begin{tabular}{|l|l|c|c|c|c|c|}
\hline \multicolumn{7}{|c|}{ Comunidad autónoma de residencia } \\
\hline r13 & $\begin{array}{l}\text { Com. de Madrid }=1,0 \text { otro } \\
\text { caso }\end{array}$ & 0,032 & 0,176 & 0 & 1 & 34.052 \\
\hline r14 & $\begin{array}{l}\text { R.de Murcia }=1,0 \text { otro } \\
\text { caso }\end{array}$ & 0,018 & 0,134 & 0 & 1 & 34.052 \\
\hline r15 & Navarra $=1,0$ otro caso & 0,029 & 0,168 & 0 & 1 & 34.052 \\
\hline r16 & País Vasco $=1,0$ otro caso & 0,056 & 0,231 & 0 & 1 & 34.052 \\
\hline r17 & La Rioja $=1,0$ otro caso & 0,018 & 0,131 & 0 & 1 & 34.052 \\
\hline r18 & Ceuta $=1,0$ otro caso & 0,008 & 0,091 & 0 & 1 & 34.052 \\
\hline r19 & Melilla $=1,0$ otro caso & 0,006 & 0,077 & 0 & 1 & 34.052 \\
\hline \multicolumn{1}{|c|}{ Otras características } & & & \\
\hline cuniprov & $\begin{array}{l}\text { Com. Uniprov. }=1,0 \text { en } \\
\text { otro caso }\end{array}$ & 0,161 & 0,368 & 0 & 1 & 34.052 \\
\hline isla & Isla $=1,0$ en otro caso & 0,035 & 0,184 & 0 & 1 & 34.052 \\
\hline ceu_meli & $\begin{array}{l}\text { Ceuta y Melilla }=1,0 \text { en } \\
\text { otro caso }\end{array}$ & 0,014 & 0,119 & 0 & 1 & 34.052 \\
\hline limfr & $\begin{array}{l}\text { Prov. límite c Francia }=1, \\
0 \text { otros }\end{array}$ & 0,106 & 0,308 & 0 & 1 & 34.052 \\
\hline limpor & $\begin{array}{l}\text { Prov. límite c. Portugal }= \\
1,0 \text { otros }\end{array}$ & 0,128 & 0,335 & 0 & 1 & 34.052 \\
\hline duracion & Número de noches & 2,619 & 5,941 & 0 & 99 & 34.052 \\
\hline
\end{tabular}

